\title{
Differential Properties of Human ALP+ Periodontal Ligament Stem Cells vs Their ALP- Counterparts
} \author{
George TJ Huang ${ }^{1,2, *}$ \\ ${ }^{1}$ Department of Bioscience Research, University of Tennessee Health Science Center, College of Dentistry, Memphis, USA \\ ${ }^{2}$ Department of Endodontics, Henry M. Goldman School of Dental Medicine, Boston University, Boston, USA \\ ${ }^{3}$ Département d'endodontie, Faculte de medicine dentaire, Université Laval, Quebec, QC, Canada \\ ${ }^{4}$ Department of Preventive Medicine, College of Medicine, Memphis University of Tennessee Health Science Center, USA \\ "Authors contributed equally.
}

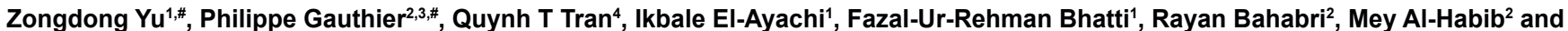

\begin{abstract}
Characterizing subpopulations of stem cells is important to understand stem cell properties. Tissue-nonspecific alkaline phosphatase (ALP) is associated with mineral tissue forming cells as well as stem cells. Information regarding ALP subpopulation of human periodontal ligament stem cells ( $h P D L S C s$ ) is limited. In the present study, we examined $\mathrm{ALP}^{+}$and ALP- $\mathrm{hPDLSC}$ subpopulations, their surface markers STRO-1 and CD146, and the expression of stemness genes at various cell passages. We found that $\mathrm{ALP}^{+}$subpopulation had higher levels of STRO-1 $(30.6 \pm 5.6 \%)$ and CD146 (90.4 $\pm 3.3 \%$ ) compared to ALP- (STRO-1: $0.5 \pm 0.1 \%$; CD146: $75.3 \pm 7.2 \%)$. ALP ${ }^{+}$cells expressed significantly higher levels of stemness associated genes, NANOG, OCT4 and SOX than ALP- cells at low cell passages of 2-3 $(p<0.05) . A^{-P^{+}}$and ALP- cells had similar osteogenic, chondrogenic and neurogenic potential while ALP-, not ALP ${ }^{+}$ cells, lacked adipogenic potential. Upon continuous culturing and passaging, ALP ${ }^{+}$continued to express higher stemness genes and STRO-1 and CD146 than ALP- cells at $\geq$ passage 19. Under conditions (over-confluence and vitamin $C$ treatment) when $\mathrm{ALP}^{+}$subpopulation was increased, the stemness gene levels of $\mathrm{ALP}^{+}$was no longer significantly higher than those in ALP- cells. In conclusion, ALP ${ }^{+}$hPDLSCs possess differential properties from their ALP-counterparts.
\end{abstract}

Keywords: Human periodontal ligament stem cells; PDLSCs; Mesenchymal stem cells; MSCs; Alkaline phosphatase; ALP; STRO1; CD146; Stemness genes; OCT4; NANOG; SOX2; Subpopulation; Osteogenic induction; BSP; RUNX2; OCN; Adipogenesis; Chondrogenesis; Neurogenesis

Abbreviations: hPDLSCs: Human Periodontal Ligament Stem Cells; PDL: Periodontal Ligament; DPSCs: Dental Pulp Stem Cells; MSC: Mesenchymal Stromal/stem Cells; ALP: Alkaline Phosphatase; CFU-F: Colony Formation Units of Fibroblastic Cells; BSP: Bone Sialophosphoprotein; OCN: Osteocalcin; RUNX2: Runt-related Transcription Factor 2; LPL: Lipoprotein Lipase; PPAR 2 : Peroxisome Proliferator-activated Receptor Gamma 2; NFM: Neurofilament M; CNPase: 2', 3'-cyclic nucleotide-3'-phosphodiesterase; GFAP: Glial Fibrillary Acidic Protein; NF1: Neurofibromin 1; Nav1.6: Sodium Channel SCN8A

\section{Introduction}

Since the isolation and characterization of human periodontal ligament stem cells (hPDLSCs) [1], these cells have been shown to have potential for regenerative purposes [2-4]. Therefore, further understanding of the PDLSC characteristics and properties is important. PDLSCs are a type of mesenchymal stromal/stem cells (MSCs) with multiple differentiation potential $[1,5,6]$. Tissue-nonspecific alkaline phosphatase (ALP) is commonly known as an early marker gene during osteogenesis and odontogenesis and it plays a role in bone matrix mineralization [7-12]. In vivo studies suggest that PDLSCs may differentiate into cementoblasts and osteoblasts $[1,13]$. Subpopulation studies of trabecular bone derived cells or bone marrow MSCs have linked the $\mathrm{ALP}^{+}$subpopulation to more differentiated osteogenic cells whereas ALP cells are more immature cells $[14,15]$. Of note, ALP is also a marker to indicate the undifferentiated state of embryonic stem cells (ESCs) [16] and induced pluripotent stem cells (iPSCs) [17] besides other pluripotency-associated genes such as NANOG, OCT4 and SOX2. The latter three have also been used to identify the stemness levels of adult stem cells and their expression levels can increase in these adult stem cells after certain treatments $[18,19]$. Therefore, to further understand the stem cell properties of PDLSCs, it is logical to ask whether $\mathrm{ALP}^{+} / \mathrm{ALP}^{-}$subpopulations in PDLSCs are associated with cell stemness levels.

STRO-1 and CD146 have been associated with MSC stemness $[20,21]$. STRO- $1^{+}$or $\mathrm{CD} 146^{+}$subpopulation from human dental pulp stem cells (DPSCs) form more colony-forming units-fibroblastic (CFUs-F) than the heterogeneous pool of DPSCs [22]. The expression of both markers tend to decrease at higher cell passages in cultures $[18,23]$. CD146-expressing cells in human bone marrow (BM) stroma are capable of establishing hematopoietic microenvironment after transplantation into heterotopic sites [21]. In the present study, we aimed to isolate and characterize $\mathrm{ALP}^{+}$hPDLSCs and examine their correlation with the expression of STRO-1, CD146, and pluripotencyassociated genes in comparison to their ALP- counterparts.

\section{Materials and Methods}

\section{Cell cultures}

PDL tissues were removed from extracted third molars $(n=27)$ of

*Corresponding author: George TJ Huang, Department of Bioscience Research, College of Dentistry, University of Tennessee Health Science Center, 875 Union Avenue, Memphis, USA, Tel: 901-448-1490; Fax: 901-448-3910; E-mail: gtjhuang@uthsc.edu

Received June 09, 2015; Accepted July 14, 2015; Published July 16, 2015

Citation: Yu Z, Gauthier P, Tran QT, El-Ayachi I, Bhatti FUR, et al. (2015) Differential Properties of Human ALP+ Periodontal Ligament Stem Cells vs Their ALP- Counterparts. J Stem Cell Res Ther 5: 292. doi:10.4172/2157-7633.1000292

Copyright: (c) 2015 Yu Z, et al This is an open-access article distributed under the terms of the Creative Commons Attribution License, which permits unrestricted use, distribution, and reproduction in any medium, provided the original author and source are credited. 
healthy patients between 14 and 30 years old in the Oral Surgery Clinics at Boston University (BU) or University of Tennessee Health Science Center (UTHSC). The patient sample collection in this study conformed to exempt protocols approved by the Institutional Review Board (IRB) of BU (\#H-28882) and UTHSC (12-01937-XM). The tissues were minced into $1 \mathrm{x} 1 \mathrm{x} 1 \mathrm{~mm}$ fragments, digested in collagenase/dispase to obtain single-cell suspensions as described previously $[1,24]$. The formation of CFU-F was observed and allowed to expand for passaging. Some cultures at passage 0 were stained for the presence of STRO- 1 . To study growth difference of PDL cells isolated either by enzyme digestion or explant outgrowth, some PDL samples were divided into two halves to isolate PDLSCs by enzyme digestion (PDLSCs-d) or explant outgrowth method (PDLSCs-o). Cells were grown in a-minimum essential medium ( $\alpha$-MEM; Life Technologies/GIBCO BRL, Gaithersburg, MD) supplemented with $10 \%$ fetal bovine serum (FBS), $2 \mathrm{mM} \mathrm{L}$-Glutamine, $100 \mu \mathrm{M}$ L-ascorbic acid-2-phosphate, and antibiotic/antimycotic agents. Human bone marrow (BM)-derived MSCs (BMMSCs) were obtained from Dr. R. S. Tuan (National Institutes of Health, Bethesda, MD) and cultured on the basis of our previous report [25]. Human jaw bone-derived MSCs (JBMSCs) were isolated as described previously [23] and grown in the same medium as for PDLSCs. For passaging, cells were split (1:3 ratio) at $\sim 80 \%$ subconfluence.

\section{Proliferation analysis}

For cell proliferation studies of PDLSC subpopulations, cells were sorted at passage 3. Original pool, $\mathrm{ALP}^{+}$and ALP- PDLSCs were then immediately plated at a density of 1,000 cells/well of 12 -well plates. Cells were then harvested and counted in a hemocytometer under the microscope at a later time point.

\section{Immunocytofluorescence analysis}

The following mouse anti-human primary antibodies were used for immunocytofluorescence. Mouse anti-human: STRO-1 IgM (Invitrogen), CD73 IgG1 (BioLegend), CD90 IgG1 (BD Pharmingen), CD105 IgG1 (eBioscience, San Diego, CA), CD146 IgG1 (Invitrogen), $\beta$ III-tubulin antibodies (Promega corp., Madison, WI), and isotype controls IgG and IgM (both from Invitrogen). Secondary antibodies included goat anti-mouse IgM or IgG1 Alexa Fluor 594 and goat antimouse IgG1 Alexa Fluor 488, all from Invitrogen.

Cells grown in chamber glass slides ( 8 wells) or in culture plates were washed and fixed with $100 \%$ ice-cold methanol for 7-10 minutes. After PBS washing, cells were blocked with $5 \%$ goat serum in PBS or in blocking buffer (32.5 mM NaCl, $3.3 \mathrm{mM} \mathrm{Na} 2 \mathrm{HPO} 4,0.76 \mathrm{mM} \mathrm{KH} 2 \mathrm{PO} 4$, $1.9 \mathrm{mM} \mathrm{NaN3}, 0.1 \%$ [w/v] bovine serum albumin (BSA), $0.2 \%(\mathrm{v} / \mathrm{v})$ Triton-X 100, 0.05\% (v/v) Tween 20, and 5\% goat serum) for $30 \mathrm{~min}$. The primary antibody was then added directly to cells and incubated for 1 hour at room temperature, washed with PBS for 3 times each 5 minutes on a rocker. After PBS wash, secondary antibody (Alexa Fluor 594 or Alexa Fluor 488) in blocking buffer was added and incubated for 1 hour at room temperature in dark. Subsequently, cell nuclei were stained with 4,6-diamidino-2-phenylindole dihydrochloride (DAPI) for 3 minutes. Images were analyzed under a fluorescence microscope.

\section{Flow cytometry}

Subconfluent cells were harvested for analysis and the antibodies used were the following: FITC-STRO-1 (Biolegend, San Diego, CA), APC-ALP (R\&D Systems, CustomerService@RnDSystems.com); PECD146; PE-Cy7-CD90; PerCP-Cy ${ }^{\mathrm{rx}}$ 5.5-CD73 (the latter three all from BD Pharmingen ${ }^{\mathrm{Tw}}$ ); V450-CD105 (BD Horizon ${ }^{\mathrm{Tw}}$ ). Relevant conjugated mouse $\operatorname{IgG}_{1}\left(\mathrm{BD}\right.$ Pharmingen ${ }^{\mathrm{Tm}}$ ) or IgM (Biolegend, San Diego, CA) were used as isotype control.

For direct single staining of cell surface antigens, cell aliquots (2-8 x $10^{5}$ cells) were washed twice with $1 \%$ BSA in phosphate buffered saline ( PBS), re-suspended in a buffer [0.1\% FBS in phosphate buffered saline (PBS)] or a blocking buffer $(10 \mu \mathrm{g} / \mathrm{mL}$ mouse IgG in PBS) for 15 minutes at room temperature, and incubated for $60 \mathrm{~min}$ at $4^{\circ} \mathrm{C}$ in the dark with conjugated PerCP Cy5.5, Cy7, APC. PE, FITC or Alexa Fluor antibodies according to the manufacture's recommendations. Cells were then washed twice and re-suspended in 1\% BSA/PBS for analysis on a flow cytometer (LSRII flowcytometer, BD Biosciences) using the FlowJo X software (BD Biosciences).

For direct quadruple staining, an anti-mouse Ig/negative control compensation polystyrene microsphere set $\left(\mathrm{AbC}^{\mathrm{m}}\right.$ Anti-Mouse Bead Kit, Invitrogen, Eugene, OR) was used for the quadruple staining according to the manufacturer's instruction. Cell aliquots of $2 \times 10^{5}$ placed in a sample tube were washed twice in a staining buffer $(1 \%$ BSA), resuspended in a blocking buffer $(10 \mu \mathrm{g} / \mathrm{mL}$ mouse IgG in PBS) and incubated for $15 \mathrm{~min}$ at room temperature. Four anti-human fluorochrome-conjugated mouse IgM or IgG antibodies -- FITCSTRO-1, APC-ALP, PE-CD146 and PE-Cy7-CD90 of appropriate dilution were all added into the cell sample tube and vortexed. After incubation for 60 minutes at $4^{\circ} \mathrm{C}$ (in the dark), cells were then washed twice and resuspended in staining buffer for analysis. The negative control compensation polystyrene microsphere set was prepared as follows. Negative beads and the $\mathrm{AbC}^{\mathrm{m}}$ anti-mouse Ig binding beads were added into empty sample tubes and vortexed. Each conjugated antibody FITC-STRO-1, APC-ALP, PE-CD146 or PE-Cy7-CD90 of the same respective dilution as for staining the cells was then added to a sample tube containing the beads and vortexed. The antibody and bead mixture was incubated at room temperature in the dark for 30 minutes and then washed with staining buffer followed by resuspending in fresh staining buffer for analysis. At the time for each flow cytometry analysis of the quadruple staining, 6 sample tubes were prepared: 1) unstained cells $\left(10^{5}\right)$, 2) FITC-STRO-1/bead mixture, 3) APC-ALP/bead mixture, 4) PE-CD146/bead mixture, 5) PE-Cy7-CD90/bead mixture and 6) cells stained with all four antibodies. Optimized fluorescence compensation settings for multicolor flow cytometric analyses were performed using a LSR II flow cytometer (BD Biosciences) and the CellQuest ProTM software (BD Biosciences).

\section{Cell sorting}

$\mathrm{ALP}^{+} / \mathrm{ALP}^{-}$cells were separated by magnetic beads cell separation (MACS Separator kits, MACS Miltenyi Biotec Inc. Auburn, CA) according to manufacturer's instruction. Cells $\left(10^{7}\right)$ were harvested and washed in cell sorting buffer (0.5\% BSA and $2 \mathrm{mM}$ EDTA in PBS, pH 7.2), centrifuged and resuspended in the blocking buffer, same as that used in FACS sorting described below, for $15 \mathrm{~min}$ at room temperature. APC-ALP antibody was then added to the cells and incubated in the dark for $60 \mathrm{~min}$ at $4^{\circ} \mathrm{C}$. Cells were then washed twice in sorting buffer and resuspended in $80 \mu \mathrm{L}$ sorting buffer. Twenty $\mu \mathrm{L}$ of Anti-APC Microbeads were then added to the cells and mixed well followed by incubation for $15 \mathrm{~min}$ at $4^{\circ} \mathrm{C}$ in the dark. Subsequently, cells were washed in buffer and resuspended in $500 \mu \mathrm{L}$ sorting buffer and loaded onto a prepared column placed on a separator. The collected cells that flowed through the column were ALP- cells. The column was then removed from the separator and placed on a collection tube. The $\mathrm{ALP}^{+}$ cells were collected from the column after adding the buffer and firmly pushing the plunger into the column.

We also applied fluorescence activated cell sorting (FACS) to sort 
$\mathrm{ALP}^{+} / \mathrm{ALP}^{-}$cells, $10^{7}$ cells were washed twice with sorting buffer $(5 \%$ FBS in culture medium), resuspended in a blocking buffer $(10 \mu \mathrm{g} / \mathrm{mL}$ mouse IgG in PBS) and incubated for $15 \mathrm{~min}$ at room temperature. Mouse anti-human ALP (APC-ALP) antibody of appropriate dilution was added into the cells and vortexed. After incubation for 60 minutes at $4^{\circ} \mathrm{C}$ (in dark), cells were washed twice in a sorting buffer (5\% FBS in cell culture medium) and resuspended in the same buffer for FACS (BD Biosciences FACSAria Cell Sorter). Sorted cells were collected in a collecting buffer (20\% FBS in culture medium), further cultured, frozen down or passaged until experimentation. We found that using FACS or MACS yielded similar results. In the present study, FACS was used for data in Figure 3C, all other data required sorting were from the MACS method.

\section{Lineage differentiation}

Osteogenic differentiation: Cells were seeded into 12-, 24or 48-well plates, grown to $\sim 70-80 \%$ confluence and incubated in differentiation medium containing $10 \mathrm{nM}$ dexamethasone, 10 $\mathrm{mM} \beta$-glycerophosphate, $50 \mu \mathrm{g} / \mathrm{ml}$ ascorbate phosphate, $10 \mathrm{nM} \mathrm{1}$, 25 dihydroxyvitamin $\mathrm{D}_{3}$ and $10 \%$ FBS for various time points and then processed for analyses. The medium was changed every 3 days. Cultures were harvested for qPCR, or fixed in 60\% isopropanol, and mineralization of extracellular matrix stained with $1 \%$ Alizarin red S (ARS) [24]. For quantitative analysis, the ARS stained cultures were dissolved in cetylpyridinium chloride (CPC) buffer (10\% CPC (w/v) in $10 \mathrm{mM}$ Sodium Phosphate Buffer, Sigma) for 1 hour. Three aliquots in $200 \mu \mathrm{L}$ of ARS/CPC extract from each well were then transferred to a 96-well reading plate and quantified by absorbance measurement at 550 $\mathrm{nm}$ by a spectrophotometer (Bio-Rad).

Adipogenic differentiation: Cells were seeded in 12- or 48well plates and grown to subconfluence as for osteo-induction, and incubated in adipogenic medium containing $1 \mu \mathrm{M}$ dexamethasone, 1 $\mu \mathrm{g} / \mathrm{ml}$ insulin, $0.5 \mathrm{mM}$ 3-isobutyl-1-methylxantine (IBMX) and $10 \%$ FBS for 6-8 weeks. The medium was changed every 3 days. Cultures were harvested for qPCR or fixed in $10 \%$ formalin for $60 \mathrm{~min}$, washed with $\mathrm{dH}_{2} \mathrm{O}$ and $60 \%$ isopropanol, and lipid droplets were stained with $0.18 \%(\mathrm{w} / \mathrm{v})$ Oil Red O reagent for $5 \mathrm{~min}$ and washed with water.

Chondrogenic differentiation: Cells were seeded into 24-well plates or into $200 \mu \mathrm{l}$ microfuge tubes and centrifuged down to form cell pellets. Cell cultures in plates or in tubes were treated for 3 weeks with chondrogenic medium, consisting of high-glucose DMEM supplemented with $100 \mathrm{nmol} / \mathrm{L}$ dexamethasone, $50 \mu \mathrm{g} / \mathrm{ml}$ ascorbic acid-2-phosphate, $100 \mu \mathrm{g} / \mathrm{ml}$ sodium pyruvate, $40 \mu \mathrm{g} / \mathrm{ml} \mathrm{l-proline,} 10$ $\mathrm{ng} / \mathrm{ml}$ recombinant human transforming growth factor- $\beta 3$ (TGF- $\beta 3$; R\&D Systems, Minneapolis, MN), and $50 \mathrm{mg} / \mathrm{ml}$ ITS-premix stock (BD Biosciences). The medium was changed every 3 days. Chondrogenic cell cultures or pellets were fixed in $4 \%$ buffered paraformaldehyde for 60 min, washed and stained with Alcian blue ( $\mathrm{pH}$ 3.0) to detect sulfated proteoglycans.

Neurogenic differentiation: Cells at subconfluence in chamber slides or in 12- or 24-well plates were stimulated by one of the two induction protocols. i) Neurogenic induction medium (NIM-1) consisted of Neurobasal A (Gibco-Invitrogen) with B27 supplement (GIBCO-BRL), $20 \mathrm{ng} / \mathrm{mL}$ epidermal growth factor (EGF) (BD Biosciences), and $40 \mathrm{ng} / \mathrm{mL}$ fibroblast growth factor (FGF) (BD Biosciences) was added to the cells and cultures incubated for 4 weeks with the medium refreshed every 3 days. Cultures were then analyzed by immunocytofluorescence for the expression of the neural

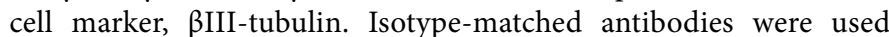

as negative controls. ii) NIM-2 consisted of $\alpha$-MEM with $10 \mathrm{ng} / \mathrm{ml}$ bFGF, $10 \mu \mathrm{M}$ forskolin (Sigma), $25 \mathrm{mM} \mathrm{KCl}, 2 \mathrm{mM}$ valproic acid and $5 \mu \mathrm{g} / \mathrm{ml}$ insulin. The cells received pre-neural induction in $\alpha$-MEM medium containing 10\% FBS and $10 \mathrm{ng} / \mathrm{ml} \mathrm{bFGF} \mathrm{(Roche)} \mathrm{for} 24 \mathrm{~h}$ [26]. Subsequently, the medium was removed, cells washed with PBS and the NIM\#2 added for up to 35 days incubation period with the medium refreshed every 3 days. Cells were monitored continually after neural induction for morphological changes and were lysed for RNA extraction or fixed for immunostaining. The control group received regular medium and harvested at the same time points as the neurogenic group.

\section{qPCR}

The cells were harvested and total RNA isolated using RNeasy mini kit (Qiagen). First strand cDNA from purified RNA was generated using the SuperScript ${ }^{\text {tm }}$ III First-Strand synthesis system for qPCR (Invitrogen). The sequence of primers is listed in Supplemental Table I. The LightCycler ${ }^{\circledR} 480$ SYBR Green I Master (Roch Diagnostic Corp., Indianapolis, IN) was used as described by the manufacturer. Briefly, cDNA template was mixed to PCR Master Mix, with forward/reverse primers and sterile distilled water to a final volume. The reaction mixture was placed in each wells of a 96well plate, covered and centrifuged at $1000 \mathrm{rpm}$ for 5 minutes at $4^{\circ} \mathrm{C}$. The plate was then placed in a LightCycler ${ }^{\circledast} 480$ II (Roch Diagnostic Corp.) and run for 40 cycles with the following thermal cycling conditions: $95^{\circ} \mathrm{C}$ for $5 \mathrm{~min}$ followed by 40 cycles of $95^{\circ} \mathrm{C}$ for $10 \mathrm{~s}$, $60^{\circ} \mathrm{C}$ for $30 \mathrm{~s}$, and $72^{\circ} \mathrm{C}$ for $10 \mathrm{sec}$. A relative quantitative analysis method was performed to quantify the relative gene expression compared to the level of the housekeeping gene glyceraldehyde-3phosphate dehydrogenase (GAPDH).

\section{Alkaline phosphatase assay}

ALP activity of PDLSCs was measured using SensoLyte pNPP ALP Assay Kit (AnaSpec, EGT Corp. Fremont, CA). Briefly, cells were lysed in assay buffer, pelleted and the supernatant collected for measurement of ALP activity. pNPP, colorimetric alkaline phosphatase substrate was applied to the sample supernatant and incubated at room temperature for $30 \mathrm{~min}$. Stop Solution was then added and the absorbance was measured at $405 \mathrm{~nm}$. The sample ALP concentration was calculated against standard ALP plots of known concentrations.

\section{Data analysis}

The strategy for the statistical analysis was to test the null hypothesis that the outcome measurements are not different among the groups of interest in each of the experiments. One way ANOVA was used to compare single factor among three or more groups. Twoway ANOVA was used to examine the effects of two independent factors and their interaction effect on the outcome. When data were collected from different donors, mixed-effects model was performed treating donor as a random effect. Shapiro-Wilk test was used to test the normality of the residuals distribution for each model. Log2 transformation was applied when the normality assumption was not met. If the main and/or interaction effect found to be statistically significant, post-hoc comparisons were examined by Tukey HSD (Honestly Significant Difference) test. Adjustment for multiple comparisons were done using Bonferroni method or Benjamini and Hochberg procedure. Values are considered statistically significant when $\mathrm{p}<0.05$. Data are reported as mean \pm SEM. All analyses were performed using JMP ${ }^{\star}$ Pro 10.0.0 and SAS 9.3 (SAS Institute Inc., Cary, NC). 
Citation: Yu Z, Gauthier P, Tran QT, El-Ayachi I, Bhatti FUR, et al. (2015) Differential Properties of Human ALP+ Periodontal Ligament Stem Cells vs Their ALP- Counterparts. J Stem Cell Res Ther 5: 292. doi:10.4172/2157-7633.1000292

Page 4 of 11

\section{Results}

\section{PDLSC properties on colony formation, population doubling and differentiation potential}

We first characterized heterogeneous populations (original pool) of PDLSCs isolated from human PDL and seeded in culture plates to observe CFU-Fs as shown in Figure 1Aa,b. These cells were also able to form CFU-F after passaging (Figure $1 \mathrm{Ac}$ ). To determine the capacity of PDLSCs to expand in vitro, population doubling (PD) studies were carried out. We divided each PDL tissue from the patients into two halves. One half underwent enzyme digestion as described in the Supplemental Materials and Methods, the other half minced into small fragments to allow the PDLSCs to out-grow into the culture plates as described in a previous report [27]. Total PD of cultured heterogeneous population of PDLSCs obtained by digestion reached $51.2 \pm 13.0$ (mean \pm SEM) ranging from 28.3 - 93.4 based on four samples from four patients (aged 20-26). PDLSCs obtained by out-growth reached the
PD of $54.8 \pm 9.3$ ranging from $24.4-72.8$. There was no statistically significant difference in the PDs between PDLSCs obtained from different methods (Supplementary Figure 1), suggesting that the two isolation methods did not cause a difference in the cell life span in cultures. In order to observe CFU formation, we then determined to use enzyme digestion method to isolate PDLSCs used for all the experiments in the present study.

At passage 0 , epithelial cell islands were frequently observed (Figure 1Ba,b). These epithelial cells are expanded from the epithelial cell rests of Malassez residing in the PDL [28]. STRO- $1^{+}$cells were normally detected in the CFU-F and tended to be located in the center of the colony (Figure 1Bc). They were also detected scattering around the epithelial islands and were not necessarily associated with CFU-Fs (Figure 1Bb, d).

PDLSCs at passages 2-3 were subjected to osteogenic, adipogenic or neurogenic induction. BMMSCs and JBMSCs were used as a
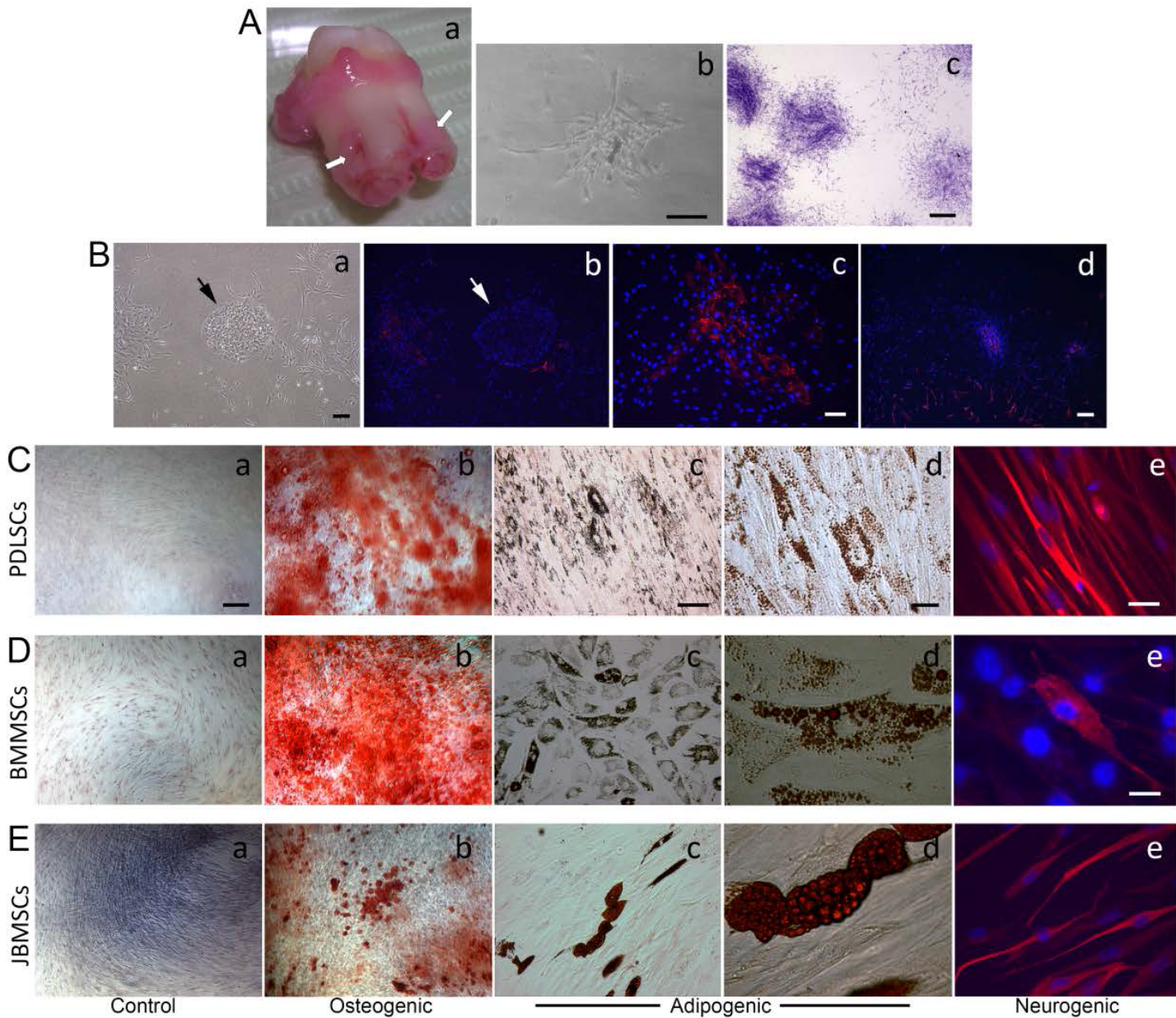

Figure 1: Isolation and characterization of heterogeneous human PDLSCs. (Aa) Image of a freshly extracted third molar. Arrows indicate PDL to be isolated. (Ab) Colony formation after initial seeding (passage 0). (Ac) PDLSC colonies formed at passage 1 fixed and stained with toluidine blue. (Ba) Initial seeding (Passage 0 ) showing an island of epithelial cells (arrow), a CFU-F (on the left) and scattered single cells. (Bb) STRO-1 staining (red fluorescence) of the culture shown in Ba. Notice the epithelia island is STRO-1 negative. The cell nuclei were stained with DAPI (blue). (Bc) STRO-1 positive in a single PDLSC CFU-F (passage 0). (Bd) STRO-1 positive PDLSC CFU-F and in scattered cells (passage 2). (C-E) Induced differentiation of PDLSCs (C), BMMSCs (D), and JBMSC (E) at passage 2-3. (Ca, Da, Ea) Controlled groups without stimulation; ( $\mathrm{Cb}, \mathrm{Db}, \mathrm{Eb})$ osteogenic induction after $\sim 6$ weeks followed by Alizarin Red stain showing mineral deposits; (c \& d of C, D, E) adipogenic induction for $\sim 6$ weeks followed by Oil-Red stain showing oil droplet-filled adipocyte-like cells; (Ce, De, Ee) neurogenic induction (in NIM-1) for 4 weeks followed by staining of $\beta$ III tubulin (red fluorescence). Scale bars: (Ab) $100 \mu \mathrm{m}$; (Ac) $1 \mathrm{~mm}$; (Ba, b) $200 \mu \mathrm{m} ;(\mathrm{Bc}) 100 \mu \mathrm{m} ;(\mathrm{a}, \mathrm{b}$ of $\mathrm{C}, \mathrm{D}, \mathrm{E}) 200 \mu \mathrm{m} ;(\mathrm{c}$ of $\mathrm{C}, \mathrm{D}, \mathrm{E}) 100$ $\mu \mathrm{m} ;(\mathrm{d}$ of $\mathrm{C}, \mathrm{d}, \mathrm{E}) 20 \mu \mathrm{m}$; (Ce, Ee) $30 \mu \mathrm{m}$; (De) $20 \mu \mathrm{m}$. 
Citation: Yu Z, Gauthier P, Tran QT, El-Ayachi I, Bhatti FUR, et al. (2015) Differential Properties of Human ALP+ Periodontal Ligament Stem Cells vs Their ALP- Counterparts. J Stem Cell Res Ther 5: 292. doi:10.4172/2157-7633.1000292

Page 5 of 11

comparison. As demonstrated in Figure 1C- E, heterogeneous PDLSCs are multipotent capable of giving rise to the three lineages following respective stimulation. Under $\sim 6$ weeks of osteogenic stimulus, cell cultures formed mineral deposits indicated by Alizarin Red stain (Figure $1 \mathrm{Cb}, \mathrm{Db}, \mathrm{Eb})$; after $\sim 6$ weeks of adipogenic stimulus, cells accumulated oil droplets revealed by Oil-red stain (Figure $1 \mathrm{Cc}, \mathrm{d}$; Dc,d; Ec,d); and after 4 weeks of neurogenic stimulus, cells were stained positively with the neural marker $\beta I I I$ tubulin (Figure $1 \mathrm{Ce}, \mathrm{De}, \mathrm{Ee}$ ).

\section{Heterogeneous PDLSCs expressed mesenchymal stem cell markers}

We further detected the expression of MSC markers STRO-1, CD73, CD90, CD105 and CD146 for PDLSCs at passage 1 using immunocytofluorescence. As shown in Figure 2A, STRO-1 and all CD markers tested were positive. In CFU-F cultures, we also counted the percentage of each MSC marker expressing cells within each colony to be as follows -- STRO-1: 20-30\%; CD73: 70\%; CD90: 100\%; CD105: $\sim 5 \%$; CD146: $\sim 51 \%$. Flow cytometry analysis after cell expansion (at passage 3) indicated the percentage of cells in the entire population expressing these markers, as depicted in Figure 2B showing high percentages of CD73, CD90 and CD146 and relatively low percentages of STRO-1 and CD105.

\section{Profiles of mesenchymal stem cell marker of $\mathrm{ALP}^{+}$PDLSC subpopulation}

We performed quadruple staining against ALP, STRO-1, CD90 and CD146 and analyzed by flow cytometry. With such an approach, we were able to obtain the following results. Cells at low passages of 1-3, $\mathrm{ALP}^{+}$cells constituted 0.1-19.7\% (Mean \pm SEM, $7.3 \pm 1.8$ ) of the total PDLSC pools based on 14 samples each from a different donor and the representative data are presented in Figure 3A. We then asked whether $\mathrm{ALP}^{+}$PDLSC subpopulation presents different levels of MSC makers compared to those in the original pool of PDLSCs or ALP cells. As shown in Figure 3B and Supplemental Table II, the percentage of cells expressing STRO- $1^{+}$in the $\mathrm{ALP}^{+}$subpopulation was 3 times more compared to the original PDLSC pool and 71 times more compared to the ALP' subpopulation.

\section{Stemness gene profiles, proliferation and differentiation capacity of PDLSC ALP ${ }^{+} /$ALP $^{-}$subpopulations at low passages}

Since $\mathrm{ALP}^{+}$PDLSCs expressed higher percentage of STRO-1, we asked whether $\mathrm{ALP}^{+}$and $\mathrm{ALP}^{-}$cells express different levels of stemness genes OCT4, NANOG and SOX2 and whether they grow at a different rate in cultures. We first used cells of low passages (p2-3) to examine these conditions. Samples from 4 donors examined, $\mathrm{ALP}^{+}$cells expressed higher levels of stemness genes (Figure $3 C)(p<0.05)$. Out of samples tested from 3 donors, two showed a slightly slower growth in $\mathrm{ALP}^{+}$than ALP cells, while that of the third donor $\mathrm{ALP}^{+}$had a faster growth; thus no statistically significant difference was shown (Figure 3D).

We next tested whether there was a difference in multi-potent differentiation capacities between $\mathrm{ALP}^{+}$and ALP subpopulations. We found that $\mathrm{ALP}^{+}$and ALP cells showed similar osteogenic, chondrogenic and neurogenic potential (Supplemental Figures 2 and 3) with minor differences in gene induction at different time points after stimulation. While no observable difference in the amount of mineral deposits in culture after osteogenesis can be noted between $\mathrm{ALP}^{+}$and ALP groups, difference in gene induction was noted. ALP and BSP expression at week 2 was not induced in ALP group, and OCN expression was not increased at week 1 in ALP- group, compared to

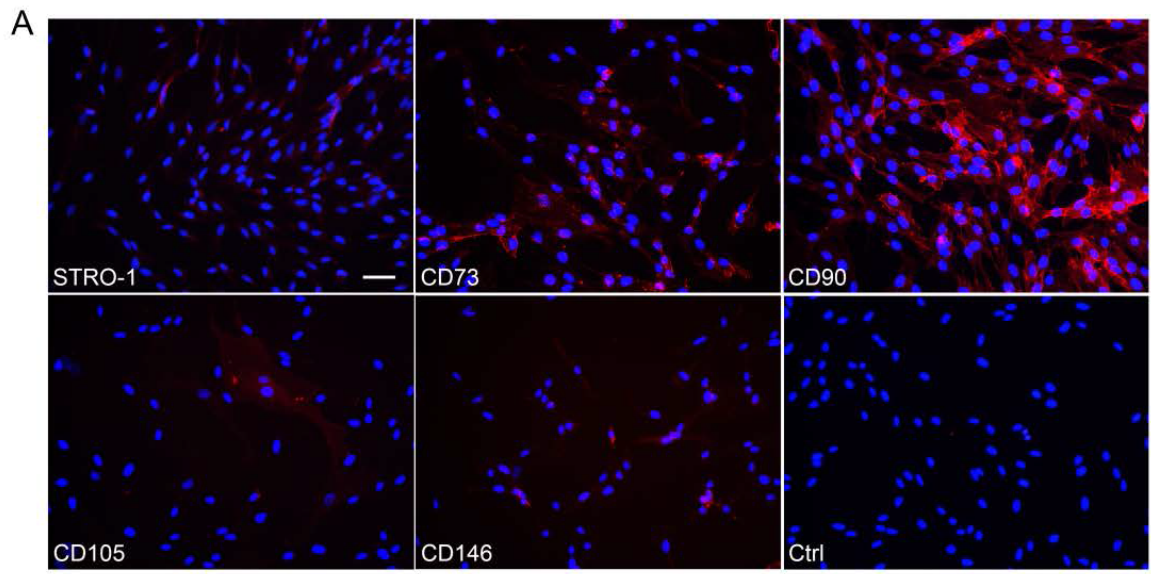

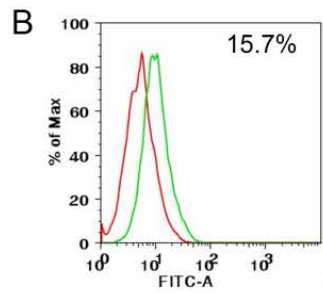

STRO-1

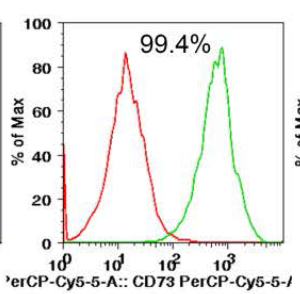

CD73

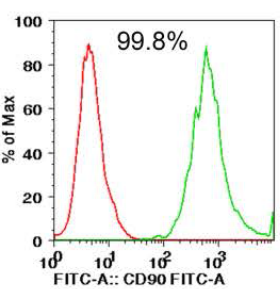

CD90

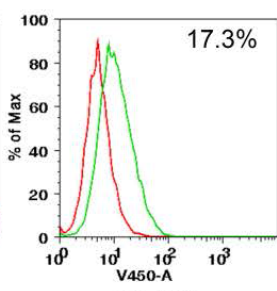

CD105

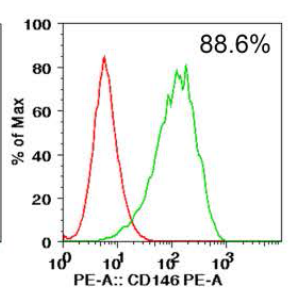

CD146

Figure 2: Marker expression by heterogeneous PDLSCs. (A) Immunocytofluorescence staining of STRO-1, CD73, CD90, CD106 and CD146. Subconfluent cells were all at passage 1. Scale bars: (A) $50 \mu \mathrm{m}$ for all marker genes and isotype control (Ctrl). (B) Flow cytometry analysis of cell surface markers and the percentage of positive cells with marker expression in the heterogeneous PDLSC population at passage 3 (red peak: control; green peak: specific antibody detection). 

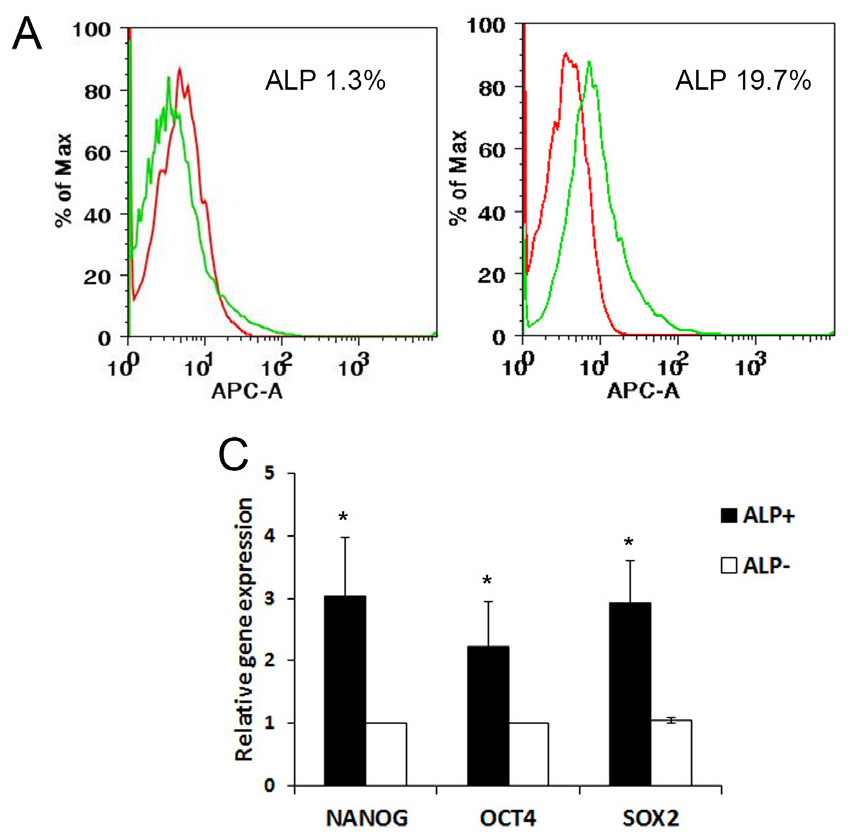
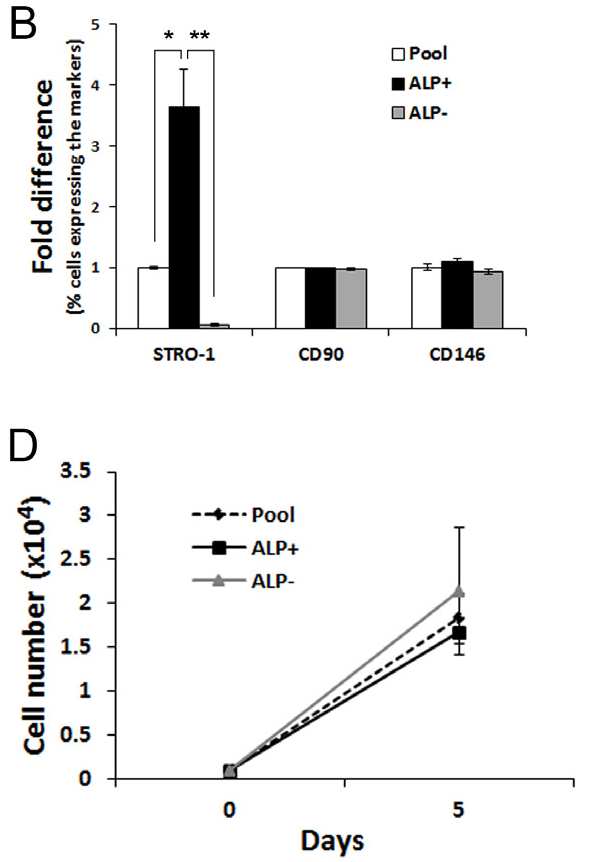

Figure 3: Characterization of the early passage ALP subpopulation of PDLSCs. (A) Flow cytometry analysis of ALP subpopulation in PDLSCs at passages 2-3. Representative data showing a wide variation of percentages of the ALP ${ }^{+}$subpopulation, with as little as $1.3 \%$ or up to $19.7 \%$. (B) Flow cytometry analysis of the quadruple staining of STRO-1, CD90, CD146 and ALP showing the levels of STRO- $1^{+}, \mathrm{CD}^{+} 0^{+}$and CD146 ${ }^{+}$cells within the subpopulations of sorted ALP ${ }^{+}$and ALP compared to the percentage of those markers in the original entire population (pool). (One-way ANOVA was performed to compare the means percentage among three populations. Tukey HSD was used to determine the pair difference. Significantly different, $\left.p<0.05^{*} ; p<0.0001^{* *} ; n=6\right)$. Original expression levels in percentage presented in supplemental Table II. Cells analyzed at passages 1-3. (C) Stemness gene expression level between ALP ${ }^{+}$and ALP- subpopulations. (Based on 4

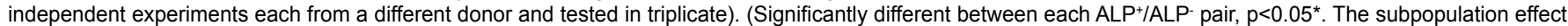
for each gene was tested using a mixed-effects model.) (D) Growth rate of the pool, ALP $^{+}$and ALP- PDLSCs. Cells were seeded at $0.1 \times 10^{4}$ per well of 12 -well plates in triplicate on day 0 and harvested/counted on day 5. Data based on 3 independent experiments ( 3 different donors), each assayed in duplicate. No significant difference was found among the subpopulation groups using a mixed-effects model $(p=0.42)$.

the $\mathrm{ALP}^{+}$group (Supplemental Figure 2B). Gene expression was not examined for chondrogenesis due to low number of $\mathrm{ALP}^{+}$cells and other cell groups had to match this cell number for comparison. For this reason, pellet culture experiments were affected. The Alcian blue staining of the monolayer cultures did not detect observable difference between $\mathrm{ALP}^{+}$and ALP- cells (Supplemental Figure 3Aa). Few successful pellet cultures showed lack of difference between $\mathrm{ALP}^{+}$and Pool cells (Supplemental Figure 3Ab). In neurogenesis, no difference can be noted from the $\beta$ III tubulin staining between groups (Supplemental Figure 3Ba). qPCR data showed that CNPase, Nav1.6 and NF1 expression was decreased or not induced in ALP- group compared to $\mathrm{ALP}^{+}$group (Supplemental Figure 3Bb).

A drastic difference between $\mathrm{ALP}^{+}$and ALP cells was found in adipogenesis that ALP' cells were incapable of undergoing adipogenic differentiation (Figure 4). $\mathrm{ALP}^{+}$cells had higher number of cells that stained positively with Oil-red than the pool and ALP' groups (Figure 4A). Additionally, adipogenic markers LPL and PPAR $\gamma$ were much more induced in $\mathrm{ALP}^{+}$cells compared to either the original pool of PDLSCs or the ALP- cells (Figure 4B).

\section{Expression of stemness genes and ALP at higher cell passages}

We then asked whether the expression of ALP, STRO-1, CD146, NANOG, OCT4 and SOX2 changes at higher cell passages. From the flow cytometry analysis of STRO-1 and CD146, both markers were higher in the $\mathrm{ALP}^{+}$subpopulation than ALP throughout passaging, particularly at later passages (Figure 5A, B). Data shown in Figure 5A are from experiments that original pool of PDLSCs were passaged and underwent quadruple staining (STRO-1, CD90, CD146 and ALP) and the STRO-1 and CD146 expression analyzed at various cell passages. Data in Figure 5B are from those that ALP- subpopulation were sorted out from the pool and continued to be passaged and analyzed with flow cytometry following quadruple staining as the above. The data showed that STRO-1 and CD146 both are higher in ALP $^{+}$than in ALP- cells (Figure $5 \mathrm{~A}, \mathrm{~B} ; \mathrm{p}<0.05$ or $\mathrm{p}<0.0001$ ). The findings indicate that both STRO-1 and CD146 are expressed at higher levels in $\mathrm{ALP}^{+}$cells either in the Pool population (Figure 5A) or in $\mathrm{ALP}^{+}$that emerged from the ALP subpopulation (Figure 5B) during passaging. Surprisingly, STRO-1 expression levels appeared to sustain the cell passaging and increased in the $\mathrm{ALP}^{+}$subpopulation even up to >passage 19. CD146 also remained high in older passages especially in the $\mathrm{ALP}^{+}$subpopulation (Figure $5 \mathrm{~B}$ ).

We further examined ALP levels in cells from low to high passages. PDLSCs from three donors were analyzed individually and data are presented in Figure 5C. The Pool group showed that ALP levels fluctuated from lower to higher passages and dwindled down after passage 20. Donor II had exceptionally high ALP levels until passage 20. The sorted $\mathrm{ALP}^{+}$subpopulation at low passages 2-3 were continuously passaged and the level dropped to a steady level and sustained until the last measurement at passages 13/14. The sorted ALP- subpopulation at passage 3 showed re-emergence of the $\mathrm{ALP}^{+}$subpopulation as cells continued to be passaged. The ALP levels increased from $0 \%$ to as high as $76 \%$ in donor II and the level decreased as passages reaching 19 . Cells from donor B, however, continued to show increased $\mathrm{ALP}^{+}$cells. 


\section{A}

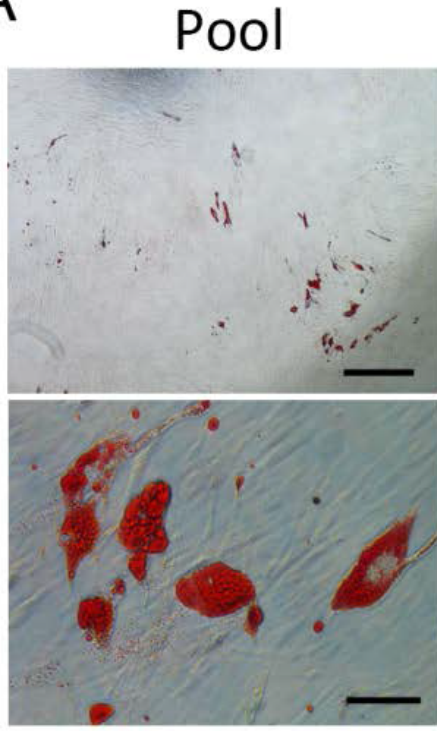

B

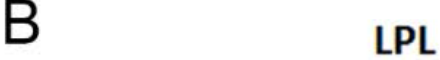

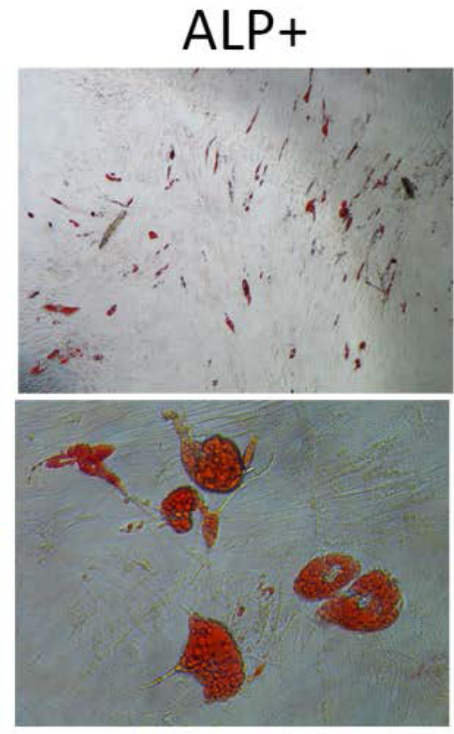

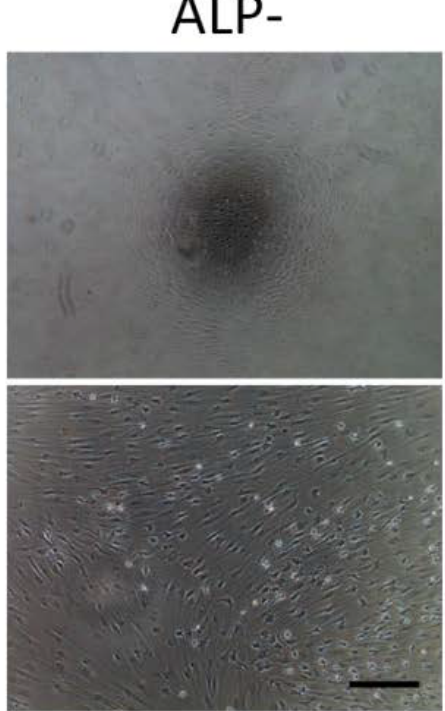

PPAR $\gamma$
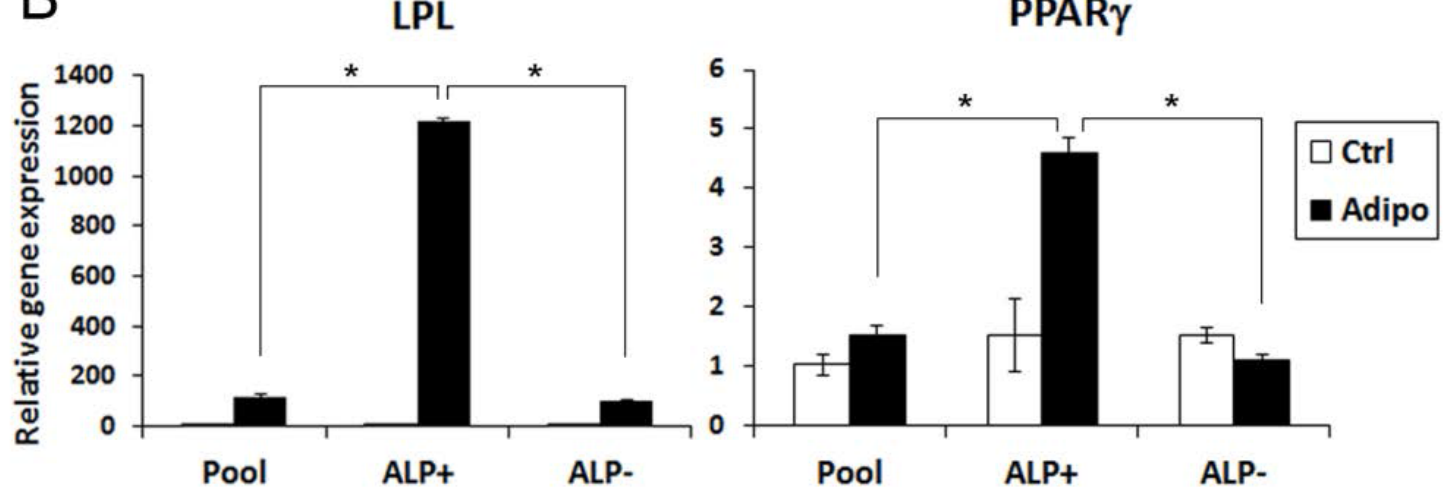

Figure 4: Adipogenic differentiation capacities of PDLSC subpopulations. Sorted PDLSCs at passages $2-3$ were immediately seeded at a density of $2 \times 10^{4}$ cells/well of 24 -well plates or $1 \times 10^{4}$ cells/well of 48 -well plates and after reaching $\sim 70 \%$ confluence they were stimulated under the adipogenic condition. (A) Adipogenic induction of cells for 8 weeks and stained with Oil Red. Scale bars: top three images, $500 \mu \mathrm{m}$; bottom left and middle images, $50 \mu \mathrm{m}$; bottom right image, $200 \mu \mathrm{m}$. (B) qPCR of $L P L$ and $P P A R Y$ in cells under adipogenic induction for 3 weeks, representative data from one donor measured in triplicate. (Significantly different, $p \leq 0.0001^{*}$. Two-way ANOVA was performed for each gene to test the subpopulation, adipogenic condition, and their interaction effects on the gene expression. Post-hoc analysis using

Tukey HSD was performed to identify the specific pairs of difference).

The stemness associated genes NANOG, OCT4 and SOX2 were also examined for their expression levels using qPCR and the data are shown in Figure 5D. Besides NANOG which appeared to sustain at the similar levels at up to passage 16, OCT4 and especially SOX2 dropped as cells reaching higher passages. To correlate the stemness associated gene levels to $\mathrm{ALP}^{+}$and $\mathrm{ALP}^{-}$subpopulations, Pool PDLSCs were sorted at passages $2 / 3$ and the sorted ALP' subpopulation were continuously passaged until passage 8 and sorted again to separate ALP $^{+} /$ALP $^{-}$ subpopulations and the stemness associated gene expression levels examined by qPCR. The data in Figure 5E show that the re-emerged $\mathrm{ALP}^{+}$population from ALP cells possessed higher stemness gene expression levels than ALP' $(\mathrm{p}<0.05$ or $\mathrm{p}<0.0001)$.

Effects of over-confluence and vitamin C treatment on ALP and stemness gene expression
All the above ALP assays were performed when PDLSCs were grown at subconfluence. We then examined ALP expression after cells were over-confluent, we found that ALP expression increased over time reaching the peak after 7 days (Figure 6A). ALP levels were significantly higher after 7 days compared to that at day 0 or 3 , while no difference was found from 7 days to 21 days. ALP levels further increased if cells were treated with vitamin C (VC) as shown in Figure 6B, especially after 3 weeks compared to the control group. We then asked whether the stemness gene expression between $\mathrm{ALP}^{+}$and ALP- cells was changed. Data in Figure 6C and D indicate that after VC treatment, the stemness gene expression levels in $\mathrm{ALP}^{+}$cells were minimally or no longer higher than those in ALP cells especially after 3 weeks of treatment (Figure 6D). Nonetheless, $\mathrm{ALP}^{+}$cells appeared to maintain the propensity to express slightly higher stemness genes, although difficult to detect the difference in some groups. ALP gene expression was higher in $\mathrm{ALP}^{+}$ cells than in ALP cells indicating that the selection of $\mathrm{ALP}^{+}$cells 
Citation: Yu Z, Gauthier P, Tran QT, El-Ayachi I, Bhatti FUR, et al. (2015) Differential Properties of Human ALP+ Periodontal Ligament Stem Cells vs Their ALP- Counterparts. J Stem Cell Res Ther 5: 292. doi:10.4172/2157-7633.1000292
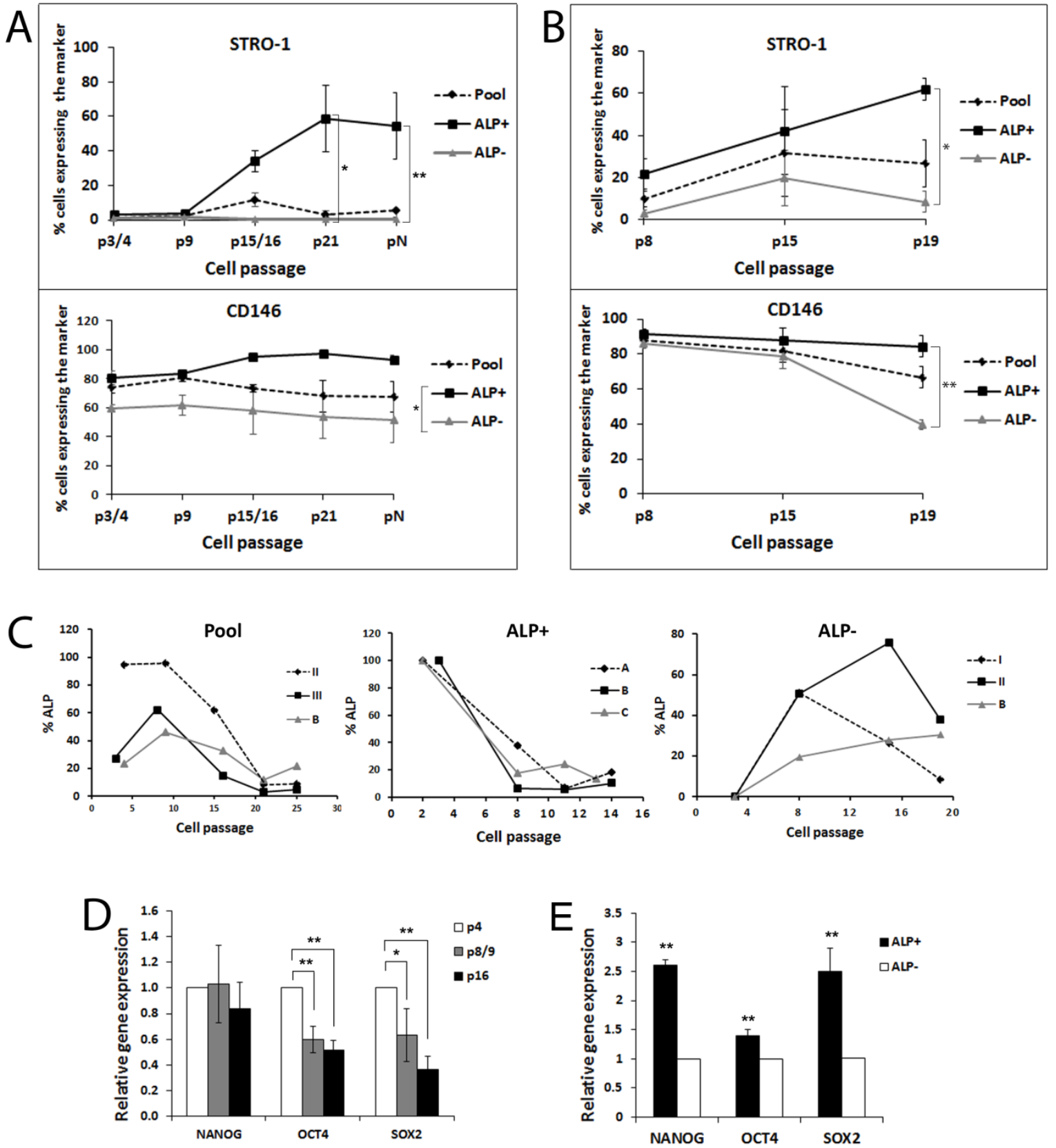

Figure 5: Expression of stemness genes by subpopulations of PDLSCs at higher passages. (A) PDLSC original pool was continuously cultured and passaged (1:3 ratio/dilution) at subconfluence and subjected to flow cytometry analysis following quadruple staining of STRO-1, CD90. CD146 and ALP. The expression of STRO-1 and CD146 of the pool and subpopulations at different passages was plotted. pN, at the cell passage axis indicates that cells were passaged at passage 8 with $1: 15$ ratio/dilution and continued to passage in this manner until reaching passage 16 (counting from passage 8 ). p3/4 or p15/16 denotes that cells were analyzed either at passage 3 or 4 , or passage 15 or 16 , respectively. Data represent mean \pm SEM of cells from three different donors. (B) PDLSC ALP- subpopulation was sorted from original pool PDLSCs at passage 3 and were continuously cultured and passaged (1:3 ratio/dilution) at subconfluence and subjected to flow cytometry analysis with the same approach as for (A). Data represent mean \pm SEM of cells from three different donors. (C) PDLSC original pools, ALP ${ }^{+}$subpopulation, and ALP- subpopulation were continuously passaged (1:3 ratio/dilution) and subjected to flow cytometry analysis to detect the percentages of the cells expressing ALP. Each graph presents data of cells from three different donors (A, B, C, I, II, II are donor codes). ALP+ subpopulation was isolated at passages 2-3 and the ALP levels were considered as $100 \%$ right after sorting. ALP- subpopulation was isolated at passage 3 and considered as $0 \%$ ALP right after sorting. (D) Expression of stemness associated genes NANOG, OCT4 and SOX2 by PDLSC pools at various passages detected by qPCR (Data represent mean \pm SEM from three different donors each assayed in triplicate). (E) Expression of stemness associated genes NANOG, OCT4 and SOX2 by PDLSC subpopulations ALP ${ }^{+}$and ALP- at passage 8 . PDLSC pools were first sorted at passages 2-3 and the ALP- subpopulation was continuously cultured and passaged until passage 8 and sorted again to separate ALP ${ }^{+}$and ALP- for qPCR analysis. (Data represent mean \pm SEM from two different donors each assayed in triplicate.) A mixed-effects model with repeated measures was performed for each gene in (A), (B), and (D) with Tukey HSD post-hoc analysis. A mixed effect model was used to test the subpopulation effect on the expression of each gene in randomly selected patients $(E)$. (Significantly different, $p<0.05^{\star} ; p<0.0001^{* *}$ ). 
A
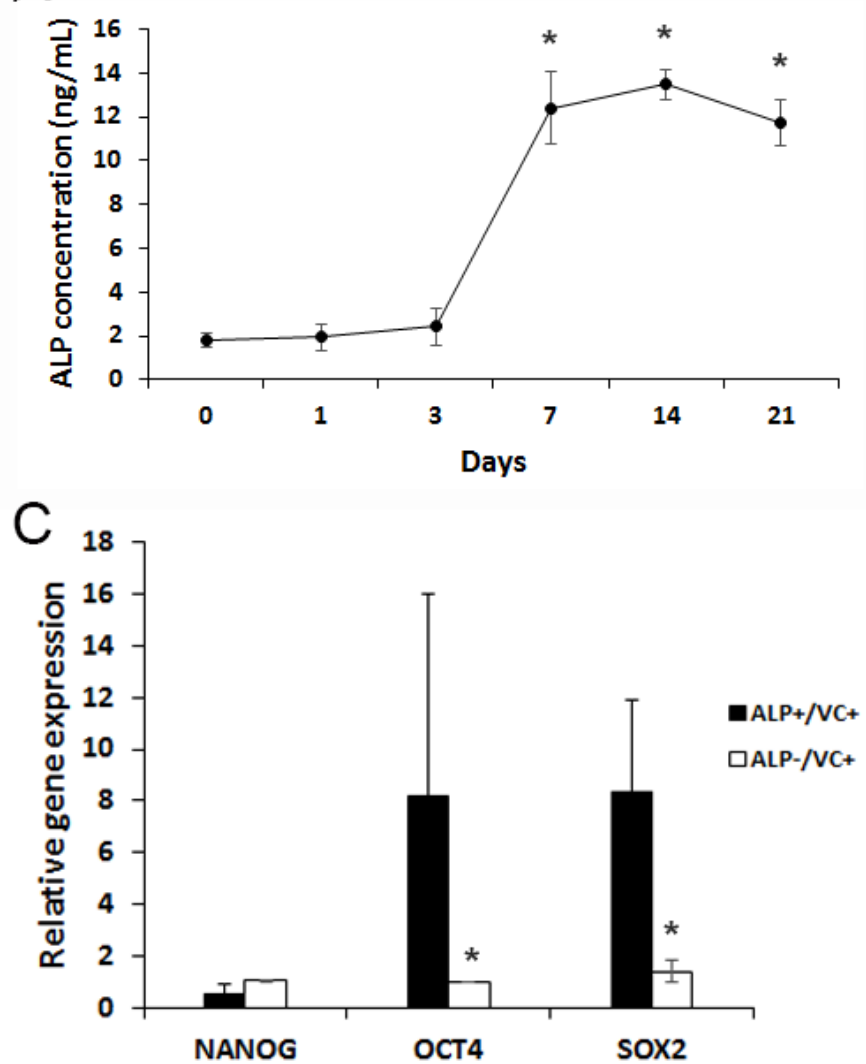

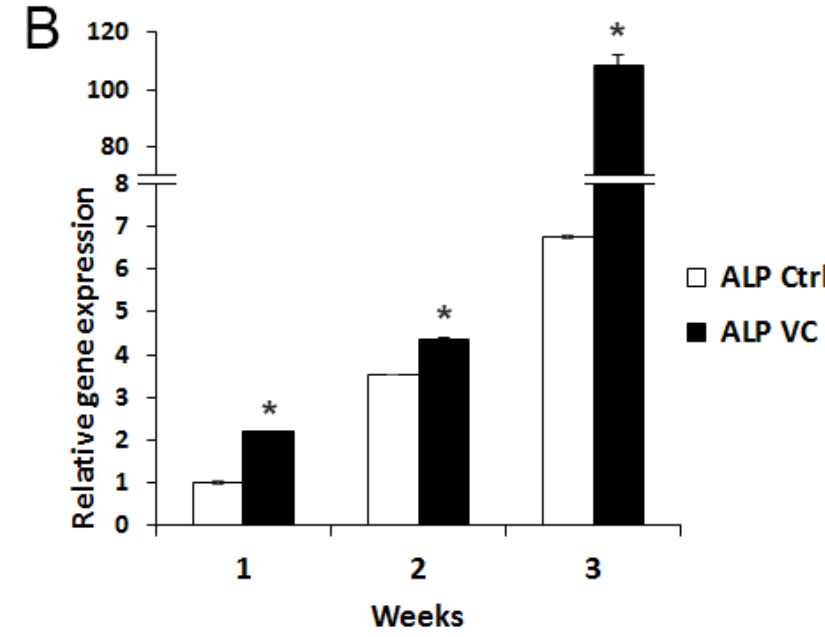

D

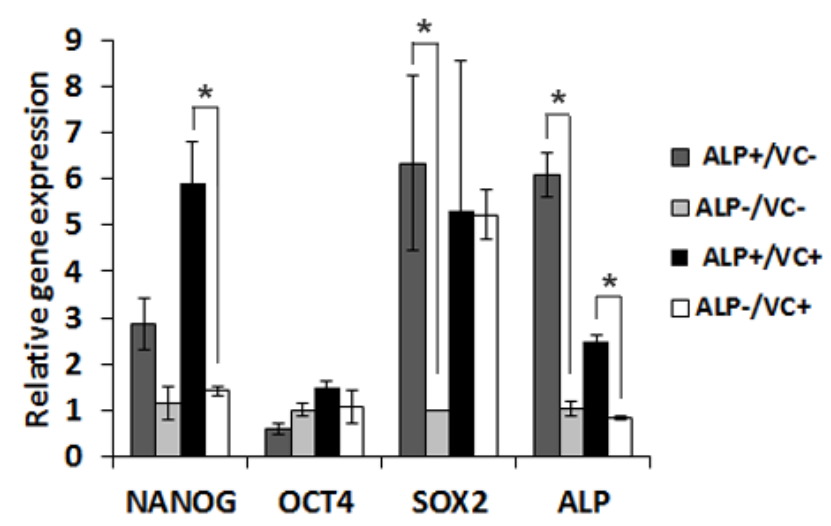

Figure 6: $\mathrm{ALP}^{+}$PDLSCs after confluence and vitamin $\mathrm{C}$ treatment. (A) PDLSCs at passages 2-4 were seeded into wells of 6-well plates and allowed to grow confluent for up to 21 days. At different time points, cells were subjected to ALP assay. Data represent mean \pm SEM from three different donors each assayed in triplicate. A mixed effect model was used to test the effect of time on protein levels. All pairwise comparisons were done and p-values were adjusted using the Tukey method. (Significantly different, $\mathrm{p} \leq 0.0001^{*}$ ). (B) PDLSCs at passage 3 were seeded in wells of 6 well-plates and vitamin C (VC) at $20 \mu \mathrm{g} / \mathrm{mL}$ was added to experimental group at $\sim 80 \%$ subconfluence. At different time points, cells were harvested and subjected to qPCR detection of ALP expression. Data represent one donor assayed in triplicate. Two sample t-tests were used and $p$-values were adjusted using Bonferroni method. (Significantly different, $p<0.0001^{*}$ ). (C) PDLSCs were cultured with VC for 1 week and sorted into $\mathrm{ALP}^{+}$and ALP- cells for RNA isolation and qPCR analysis of the stemness gene expression. Data represent mean \pm SEM from three different donors each assayed in triplicate. (The qPCR data values were very low in these cells and some of them were below detectable levels). A mixed effect model was used to test the subpopulation effect on the expression of each gene in randomly selected donors. The p-values were adjusted using the Benjamini-Hochberg procedure. (Significantly different, $p=0.045^{*}$ ). (D) PDLSCs were cultured with or without VC for 3 weeks and cells were sorted into ALP+ and ALP- cells for RNA isolation and qPCR analysis. Data represent one donor assayed in triplicate. (Significantly different, $\left.p \leq 0.05^{\star}\right)$. (70-99\% of cells were ALP ${ }^{+}$after sorting in C, D). PDLSCs were grown in medium absent of L-ascorbic acid 2-phospate for the VC experiments. A linear model with subpopulation as the main effect (ALP+ and ALP-) was used for each gene with or without VC. $\mathrm{P}$-values were adjusted using the false discovery rate procedure.

correlated to their gene levels.

\section{Discussion}

Our present study has identified several key features of hPDLSCs in vitro: i) ALP expression is associated with higher levels of stemness gene expression. ii) Both $\mathrm{ALP}^{+}$and ALP- cells are multipotent except that ALP- cells lack adipogenic potential. iii) $\mathrm{ALP}^{+}$cells maintain higher levels of STRO-1, CD146 and NANOG expression than ALP cells in cultures even after a high number of passaging.

The maintenance of stemness of cultured adult stem cells has been studied from various perspectives. One difficulty for such studies is the variation among subtypes of MSCs and the lack of a set of universal stemness markers to identify them. Subtypes of MSCs in different mesenchymal tissues are subtly different in their lineage commitment, multi-lineage differentiation potential and in vitro proliferative capacity [6]. Another issue is cell passaging of the in vitro study. Many assays are difficult to perform to study subpopulations at passage 0 due to low cell numbers. We used low passages 2-3 to perform the subpopulation separation. For cell sorting, we needed $0.5-1 \times 10^{7}$ of cells rendering the use of passage 0 technically prohibitive. Another difficulty is the frequent low percentage of $\mathrm{ALP}^{+}$cells among the cell populations at low passages or subconfluence.

Treating MSCs such as stem cells from apical papilla (SCAP), another type of dental stem cells, with growth factors such as bFGF increases the expression of STRO-1, NANOG, OCT4, SOX2 and REX1 and increases the proliferation while down-regulating the 
differentiation [19]. Such enhanced expression of stemness genes and inhibition of cell differentiation in vitro is considered a boost of stem cell stemness. On the other hand, treating DPSCs with small molecules such as $\mathrm{SC} 1$ and rapamycin decreased cell proliferation although the expression of the stemness genes NANOG, OCT4 and SOX2 is upregulated and the differentiation down-regulated [18]. There appears to have contradictory findings in the relationship between stemness and proliferation rate in vitro. The issue lies in the definition of selfrenewal and proliferation when cells are grown in cultures. Stem cell renewal and progenitor cell clonal amplification is a different stage of cell status, although both go through cell cycle/division. Sacchetti et al. identified a subpopulation CD146 $6^{+}$BMMSCs that are modulated in opposite ways [21]. bFGF increases cell proliferation and attenuates the stem cell phenotype, while TGF- $\beta$ inhibits proliferation and preserves or enhances the stemness. Their findings suggest that enhanced proliferation pushes BMMSCs toward more differentiated progenitor cells rather than maintained as stem cells which manifest as slow proliferating in cultures. The association of $\mathrm{ALP}^{+}$PDLSCs with higher expression of STRO-1, CD146, NANOG, OCT4 and SOX2 suggests that these are a more immature phenotype than the ALP- PDLSCs. ALP ${ }^{+}$ cells do not have a higher proliferation rate, some cases lower, than ALP cells which may indicate a slight link to the situation of the small molecule treated DPSCs or TGF- $\beta$ treated BMMSCs. Because ALP is a marker for the undifferentiated state of pluripotent stem cells $[16,17]$, our findings on the association of PDLSC ALP ${ }^{+}$subpopulation with the higher expression of stemness genes indicate that such phenomenon also applies to adult stem cells in the case of PDLSCs. Additionally, $\mathrm{ALP}^{+}$cells maintained higher expression of STRO-1, NANOG, OCT4 and SOX2 than ALP cells at high passages, which also supports the stemness status of $\mathrm{ALP}^{+}$subpopulation. This is in contrast to BMMSCs that ALP cells have more multipotential capacity and express higher pluripotent marker genes REX1 and NANOG than $\mathrm{ALP}^{+}$[15].

Regarding that lack of adipogenic potential of ALP- PDLSCs, two mechanisms may be involved. One is that ALP' subpopulation is less immature than $\mathrm{ALP}^{+}$. BMMSCs lose chondrogenesis and adipogenesis after passaging whereas osteogenesis is the last differentiation potency to lose [29]. The other is that ALP is involved in the control of intracellular lipid accumulation in preadipocyte maturation [30,31], therefore, absence of ALP may prevent formation of lipid in cells.

Our studies also showed that $\mathrm{ALP}^{+}$is transient in the pool of PDLSCs evidenced by the finding that the percentage of $\mathrm{ALP}^{+}$cells in the sorted $\mathrm{ALP}^{+}$subpopulation came down after cell passaging while the $\mathrm{ALP}^{+}$subpopulation also re-emerged from sorted ALP- cells. This $\mathrm{ALP}^{+}$subpopulation either in the original PDLSC pool or emerged from ALP subpopulation at low or high passages expressed higher levels of stemness genes than the ALP- subpopulation. At low passages 1-3, $\mathrm{ALP}^{+}$cells constituted 0.1-19.7\% of the total PDLSC pools based on 14 samples each from a different donor. There was a cell sample from another donor expressing ALP as high as $94.5 \%$ measured at passage 4 (Figure 5C, Pool Group, donor II) and the ALP levels stayed high until passage 21 . The sorted ALP- subpopulation from this sample also showed a drastic re-emergence of $\mathrm{ALP}^{+}$subpopulation upon further cell culturing and passaging (Figure 5C, ALP- Group, donor II). This sample was exceptional among all samples we tested, indicating that potentially, although less common, PDLSCs from some donors may contain very high percentage of $\mathrm{ALP}^{+}$cells.

The re-emergence of $\mathrm{ALP}^{+}$from ALP cells and their expression of higher level of stemness genes than ALP' cells indicates that PLDSCs appear to contain subpopulations that remain immature in cultures after long-term passaging and ALP is a marker for such type of subpopulations. Recent studies have shown that human PDLSCs contain subpopulation connexin $43^{+}$cells that are highly potent capable of forming teratomas in vivo with tissues representing all three germ layers [32]. This could also be due to an in vitro culturing phenomenon. Pierantozzi et al. [33] have shown that NANOG expression in MSCs increased after culturing. Several findings in our present studies may explain these phenomena: i) $\mathrm{ALP}^{+}$cells are always associated with higher levels of NANOG, OCT4 and SOX2 than ALP- cells (Figure 3C, 5E); ii) NANOG appeared to sustain high levels after passaging (Figure 5D), and iii) PDLSCs maintain high levels of STRO-1, CD146 at high passages. It is likely that PDLSCs maintain their stem/progenitor status by having subpopulation of cells capable of reversing to or remaining at the immature stage. Another possibility is the cell cycle-dependent ALP expression identified in the studies of human bone cells [34]. However, the relationship of this cell cycle dependence to ALP association with stemness is not clear.

When PDLSCs cells were grown in conditions that favor cell differentiation such as over-confluence or VC treatment, $\mathrm{ALP}^{+}$ subpopulation and its levels increased, which is similar to bone cells [34]. Interestingly, despite what was anticipated that under differentiation conducive conditions, stemness genes expressed by $\mathrm{ALP}^{+}$PDLSCs seemed to retain some potential to be higher than the ALP subpopulation, albeit minimal; unlike bone derived cells that $\mathrm{ALP}^{+}$subpopulation is more associated with more differentiated state than ALP- counterparts [14]. In conclusion, our present studies revealed the dynamic ALP expression and its relationship to the stemness properties of the PDLSC subpopulations. Further studies are needed to understand the roles of ALP association with stemness in the PDLSC subpopulation and this relationship to ALP association with other commitment such as osteogenesis or cementogenesis.

\section{Authors' Contributions}

$\mathrm{ZY}$ and PG designed and performed the experimental work, acquired and assembled the data, and drafted the manuscript. QTT analyzed, interpreted the data, performed statistical analysis, and wrote part of the manuscript. IEA, FURB, RB, and MAH performed some of the experimental work and assembled data. GTJH conceived, designed, performed experimental works and supervised the overall project, analyzed and interpreted the data and finalized the manuscript. All authors have read and approved the manuscript for publication.

\section{Acknowledgments}

This work was supported in part by a grant from the National Institutes of Health R01 DE019156 (G.T.-J.H.), and by an Endodontic Research Grant from American Association of Endodontists Foundation (G.T.-J.H.).

\section{References}

1. Seo BM, Miura M, Gronthos S, Bartold PM, Batouli S, et al. (2004) Investigation of multipotent postnatal stem cells from human periodontal ligament. Lancet 364: 149-155. [Pubmed]

2. Liu Y, Zheng Y, Ding G, Fang D, Zhang C, et al. (2008) Periodontal ligament stem cell-mediated treatment for periodontitis in miniature swine. Stem Cells 26: 1065-1073. [Pubmed]

3. Ding G, Liu Y, Wang W, Wei F, Liu D, et al. (2010) Allogeneic periodonta ligament stem cell therapy for periodontitis in swine. Stem Cells 28: 1829-1838. [Pubmed]

4. Menicanin D, Mrozik KM, Wada N, Marino V, Shi S, et al. (2014) Periodontalligament-derived stem cells exhibit the capacity for long-term survival, selfrenewal, and regeneration of multiple tissue types in vivo. Stem Cells Dev 23 1001-1011. [Pubmed]

5. Gay IC, Chen S, MacDougall M (2007) Isolation and characterization of 
Citation: Yu Z, Gauthier P, Tran QT, El-Ayachi I, Bhatti FUR, et al. (2015) Differential Properties of Human ALP+ Periodontal Ligament Stem Cells vs Their ALP- Counterparts. J Stem Cell Res Ther 5: 292. doi:10.4172/2157-7633.1000292

Page 11 of 11

multipotent human periodontal ligament stem cells. Orthod Craniofac Res 10: 149-160. [Pubmed]

6. Huang GT, Gronthos S, Shi S (2009) Mesenchymal stem cells derived from dental tissues vs. those from other sources: their biology and role in regenerative medicine. J Dent Res 88: 792-806. [Pubmed]

7. Tsai MT, Li WJ, Tuan RS, Chang WH (2009) Modulation of osteogenesis in human mesenchymal stem cells by specific pulsed electromagnetic field stimulation. J Orthop Res 27: 1169-1174. [Pubmed]

8. Hessle L, Johnson KA, Anderson HC, Narisawa S, Sali A, et al. (2002) Tissuenonspecific alkaline phosphatase and plasma cell membrane glycoprotein-1 are central antagonistic regulators of bone mineralization. Proc Natl Acad Sci U S A 99: 9445-9449. [Pubmed]

9. Liu J, Jin T, Ritchie HH, Smith AJ, Clarkson BH (2005) In vitro differentiation and mineralization of human dental pulp cells induced by dentin extract. In Vitro Cell Dev Biol Anim 41: 232-238. [Pubmed]

10. Viereck V, Siggelkow H, Tauber S, Raddatz D, Schutze N, et al. (2002) Differential regulation of Cbfa1/Runx2 and osteocalcin gene expression by vitamin-D3, dexamethasone, and local growth factors in primary human osteoblasts. J Cell Biochem 86: 348-356. [Pubmed]

11. Komaki M, Iwasaki K, Arzate H, Narayanan AS, Izumi Y, et al. (2012) Cementum protein 1 (CEMP1) induces a cementoblastic phenotype and reduces osteoblastic differentiation in periodontal ligament cells. J Cell Physiol 227: 649-657. [Pubmed]

12. Beertsen W, VandenBos T, Everts $V$ (1999) Root development in mice lacking functional tissue non-specific alkaline phosphatase gene: inhibition of acellular cementum formation. J Dent Res 78: 1221-1229. [Pubmed]

13. Chen SC, Marino V, Gronthos S, Bartold PM (2006) Location of putative stem cells in human periodontal ligament. J Periodontal Res 41: 547-553. [Pubmed]

14. Gronthos S, Zannettino AC, Graves SE, Ohta S, Hay SJ, et al. (1999) Differentia cell surface expression of the STRO-1 and alkaline phosphatase antigens on discrete developmental stages in primary cultures of human bone cells. J Bone Miner Res 14: 47-56. [Pubmed]

15. Kim YH, Yoon DS, Kim HO, Lee JW (2012) Characterization of differen subpopulations from bone marrow-derived mesenchymal stromal cells by alkaline phosphatase expression. Stem Cells Dev 21: 2958-2968. [Pubmed]

16. Thomson JA, Itskovitz-Eldor J, Shapiro SS, Waknitz MA, Swiergiel JJ, et al (1998) Embryonic stem cell lines derived from human blastocysts. Science 282: 1145-1147. [Pubmed]

17. Takahashi K, Tanabe K, Ohnuki M, Narita M, Ichisaka T, et al. (2007) Induction of pluripotent stem cells from adult human fibroblasts by defined factors. Cell 131: 861-872. [Pubmed]

18. Al-Habib M, Yu Z, Huang GT (2013) Small molecules affect human dental pulp stem cell properties via multiple signaling pathways. Stem Cells Dev 22: 2402 2413. [Pubmed]

19. Wu J, Huang GT, He W, Wang P, Tong Z, et al. (2012) Basic fibroblast growth factor enhances stemness of human stem cells from the apical papilla. J Endod 38: 614-622. [Pubmed]

20. Shi S, Gronthos S: Bone marrow mesenchymal stem cells. In Stem cells in craniofacial development and regeneration. 1st edition. Edited by Huang GT-J,
Thesleff I. Hoboken, New Jersey: Wiley-Blackwell; 2013: 223-239. [Pubmed]

21. Sacchetti B, Funari A, Michienzi S, Di Cesare S, Piersanti S, et al. (2007) Self-renewing osteoprogenitors in bone marrow sinusoids can organize a hematopoietic microenvironment. Cell 131: 324-336. [Pubmed]

22. Shi S, Gronthos S (2003) Perivascular niche of postnatal mesenchymal stem cells in human bone marrow and dental pulp. J Bone Miner Res 18: 696-704 [Pubmed]

23. Liao J, Al Shahrani M, Al-Habib M, Tanaka T, Huang GT (2011) Cells isolated from inflamed periapical tissue express mesenchymal stem cell markers and are highly osteogenic. J Endod 37: 1217-1224. [Pubmed]

24. Huang GT, Yamaza T, Shea LD, Djouad F, Kuhn NZ, et al. (2010) Stem/ progenitor cell-mediated de novo regeneration of dental pulp with newly deposited continuous layer of dentin in an in vivo model. Tissue Eng Part A 16: 605-615. [Pubmed]

25. Sonoyama W, Liu Y, Yamaza T, Tuan RS, Wang S, et al. (2008) Characterization of the apical papilla and its residing stem cells from human immature permanent teeth: a pilot study. J Endod 34: 166-171. [Pubmed]

26. Ebrahimi B, Yaghoobi MM, Kamal-abadi AM, Raoof M (2011) Human denta pulp stem cells express many pluripotency regulators and differentiate into neuronal cells. Neural regeneration research 6: 2666-2672.

27. Huang GT, Sonoyama W, Chen J, Park SH (2006) In vitro characterization of human dental pulp cells: various isolation methods and culturing environments. Cell Tissue Res 324: 225-236. [Pubmed]

28. Sonoyama W, Seo BM, Yamaza T, Shi S (2007) Human Hertwig's epithelial root sheath cells play crucial roles in cementum formation. J Dent Res 86: 594-599. [Pubmed]

29. DiGirolamo CM, Stokes D, Colter D, Phinney DG, Class R, et al. (1999) Propagation and senescence of human marrow stromal cells in culture: a simple colony-forming assay identifies samples with the greatest potential to propagate and differentiate. Br J Haematol 107: 275-281. [Pubmed]

30. Ali AT, Penny CB, Paiker JE, Psaras G, Ikram F, et al. (2006) The relationship between alkaline phosphatase activity and intracellular lipid accumulation in murine 3T3-L1 cells and human preadipocytes. Anal Biochem 354: 247-254. [Pubmed]

31. Ali AT, Ferris WF, Penny CB, Van der Merwe M-T, Jacobson BF, et al. (2013) Lipid accumulation and alkaline phosphatase activity in human preadipocytes isolated from different body fat depots. Journal of Endocrinology, Metabolism and Diabetes of South Africa 18: 58-64. [Pubmed]

32. Pelaez D, Huang CY, Cheung HS (2013) Isolation of pluripotent neural crestderived stem cells from adult human tissues by connexin-43 enrichment. Stem Cells Dev 22: 2906-2914. [Pubmed]

33. Pierantozzi E, Gava B, Manini I, Roviello F, Marotta G, et al. (2011) Pluripotency regulators in human mesenchymal stem cells: expression of NANOG but not of OCT-4 and SOX-2. Stem Cells Dev 20: 915-923. [Pubmed]

34. Fedarko NS, Bianco P, Vetter U, Robey PG (1990) Human bone cell enzyme expression and cellular heterogeneity: correlation of alkaline phosphatase enzyme activity with cell cycle. J Cell Physiol 144: 115-121. [Pubmed] 